\title{
Human TOP1 residues implicated in species specificity of HIV-1 infection are required for interaction with BTBD2, and RNAi of BTBD2 in old world monkey and human cells increases permissiveness to HIV-1 infection
}

Bharat Khurana', Lei Zhuang ${ }^{2}$, Prasun K Moitra², Tzanko S Stantchev ${ }^{3}$, Christopher C Broder ${ }^{3}$, Mary Lou Cutler ${ }^{1,4^{*}+}$, Peter D'Arpa ${ }^{1,2+}$

\begin{abstract}
Background: Host determinants of HIV-1 viral tropism include factors from producer cells that affect the efficiency of productive infection and factors in target cells that block infection after viral entry. TRIM5 $\alpha$ restricts HIV-1 infection at an early post-entry step through a mechanism associated with rapid disassembly of the retroviral capsid. Topoisomerase I (TOP1) appears to play a role in HIV-1 viral tropism by incorporating into or otherwise modulating virions affecting the efficiency of a post-entry step, as the expression of human TOP1 in African Green Monkey (AGM) virion-producing cells increased the infectivity of progeny virions by five-fold. This infectivity enhancement required human TOP1 residues 236 and 237 as their replacement with the AGM counterpart residues abolished the infectivity enhancement. Our previous studies showed that TOP1 interacts with BTBD1 and BTBD2, two proteins which co-localize with the TRIM5 $\alpha$ splice variant TRIM5 $\delta$ in cytoplasmic bodies. Because BTBD1 and BTBD2 interact with one HIV-1 viral tropism factor, TOP1, and co-localize with a splice variant of another, we investigated the potential involvement of BTBD1 and BTBD2 in HIV-1 restriction.
\end{abstract}

Results: We show that the interaction of BTBD1 and BTBD2 with TOP1 requires hu-TOP1 residues 236 and 237, the same residues required to enhance the infectivity of progeny virions when hu-TOP1 is expressed in AGM producer cells. Additionally, interference with the expression of BTBD2 in AGM and human 293T target cells increased their permissiveness to HIV-1 infection two- to three-fold.

Conclusions: These results do not exclude the possibility that BTBD2 may modestly restrict HIV-1 infection via colocation with TRIM5 variants in cytoplasmic bodies.

\section{Background}

Upon entry into target cells, retroviruses undergo several transformations to establish a productive infection which include uncoating of the viral core, reverse transcription, nuclear access, and integration of the viral DNA into the host genome [1,2]. Factor(s) incorporated

\footnotetext{
* Correspondence: mcutler@usuhs.edu

+ Contributed equally

'Department of Pathology, Uniformed Services University of the Health

Sciences, 4301 Jones Bridge Road, Bethesda, MD (20814) USA

Full list of author information is available at the end of the article
}

into HIV-1 virions from producer cells and factor(s) present in target cells determine viral tropism [3-10].

Topoisomerase I (TOP1) activity has been found to be associated with HIV virions [11], and the species of TOP1 expressed in virion producer cells has been reported to significantly influence viral infectivity: HIV-1 virions produced by African Green Monkey (AGM) cells were $85-90 \%$ less infective to human cells as compared to virions produced by human cells [7]. Shoya et al. reported that expression of human-TOP1, but not AGM-TOP1, in HIV-1-producing AGM cells 
increased the infectivity of progeny virions about fivefold [7]. This enhancement to the infectivity of HIV-1 virions provided by the expression of $h u$-TOP1 in AGM cells was dependent on $h u$-TOP1 residues E236 and N237, as replacement of these residues with their AGM counterparts abolished the activity enhancement. The infectivity enhancement was associated with a four-fold greater copy number of HIV-1 DNA in target cells [7]. In contrast to Old World monkey producer cells, in human producer cells $(293 \mathrm{~T})$ the expression of $h u$-TOP1 only slightly increased viral infectivity. Also, expression of AGM TOP1, or $h u$ TOP1 with residues 236 and 237 replaced with the AGM counterpart residues (i.e., E236D/N237S), in human producer cells caused virions to have four-fold less infectivity [7].

TRIM5 $\alpha$ is a major factor that restricts HIV-1 infection of Old World monkey cells, and expression of rhesus monkey TRIM5 $\alpha$ in human cells confers potent resistance to HIV-1 infection [8]. Conversely, interference with TRIM5 $\alpha$ expression in Old World monkey cells relieves the block to HIV-1 infection [8]. The TRIM family of proteins contains a tripartite motif that includes RING, B-box 2 and coiled-coil-(cc) domains. Many TRIM proteins, including TRIM5 $\alpha$, assemble into cytoplasmic structures [12]. We previously reported that a non-restricting splice variant of TRIM5, TRIM5 $\delta$, localizes to cytoplasmic bodies together with BTBD1 and BTBD2. BTBD1 and BTBD2 proteins interact with TOP1, share $80 \%$ amino acid sequence identity with each other, and contain a BTB/ POZ domain and kelch-like and PHR-like regions $[13,14]$. The BTBD/POZ domain mediates homo- and hetero-dimerization and some BTB domains bind the Cul3 ubiquitin ligase and select substrates for ubiquitylation [15-18]. The kelch repeat is a $\beta$-propeller structure that appears in numerous proteins as a proteinprotein interaction site. Our observations that the BTBD1 and BTBD2 proteins physically interact with one HIV-1 restriction factor, TOP1, and co-localize with a splice variant of TRIM5 $\alpha$ prompted us to investigate the potential involvement of BTBD1 and BTBD2 in restricting HIV infection.

Here we show that the same two $h u$-TOP1 residues required for the enhancement of the infectivity of progeny virions when $h u$-TOP1 is expressed in AGM producer cells are also required for $h u$-TOP1 to bind BTBD1 and BTBD2. We also show that interference with the expression of BTBD2 modestly increases HIV-1 infection in both non-permissive AGM cells and permissive 293T cells. These two observations together suggest that BTBD2 may be linked to restriction of HIV-1 infectivity.

\section{Methods}

\section{Cell Culture}

African green monkey (COS-1), human adenocarcinoma HeLa, and large $\mathrm{T}$ antigen expressing human embryonic kidney (293T) cell lines were maintained in Dulbeco's MEM (DMEM) supplemented with $2 \mathrm{mM} \mathrm{L-Glutamine,}$ $10 \%$ fetal bovine serum, and penicillin/streptomycin.

\section{TOP1 constructs, yeast two-hybrid and GST-pulldown}

The amino terminus of $h u$-TOP1 was fused to the Tap tag [19], producing TAP-TOP $\mathrm{E} 236 / \mathrm{N} 237$ or TAP$\mathrm{TOP}_{\mathrm{D} 236 / \mathrm{S} 237}$. These plasmids were transiently transfected into HeLa cells, and the expressed TAP-TOP1 proteins were purified and used to determine that the amino acid replacements had no effect on DNA relaxation activity (Data not shown). Two hybrid and GSTpulldown assays were performed as previously described and in the legend to Figure 1 [14].

\section{Immunofluorescence}

Cells on coverslips were fixed with $4 \%$ paraformaldehyde for $10 \mathrm{~min}$ on ice and treated with $0.1 \%$ Triton X-100 for 10 minutes on ice. Fixed cells were blocked with PBS containing 3\% BSA for 1 hour at room temperature before incubation with primary antibodies. Secondary antibody incubations were at room temperature for 45 min. For detection of cytoplasmic bodies, cells were reacted with rabbit anti-BTBD1 peptide antibody [13] followed by Alexa Fluor 568 goat anti-rabbit IgG (Molecular Probes). Coverslips were mounted using Prolong Antifade kit (Invitrogen, Carlsbad CA). Fluorescence images were obtained using an Olympus IX70 inverted fluorescence microscope equipped with a Princeton Instrument cooled CCD camera integrated with Scanalytics IP-Lab software. For quantification of BTBD1/D2 cytoplasmic bodies, the acquired images were analyzed using TIFFany 3 (Caffeine Software) and the cytoplasmic bodies were quantified using Mathematica software (Wolfram research).

\section{BTBD1 and BTBD2 shRNA constructs}

The pSIREN-RetroQ vector system (Clontech, Palo Alto CA) was used to produce BTBD1 and BTBD2 specific short hairpin shRNA (under the control of the human U6 promoter). Target sequences within both BTBD1 (NM_025238) and BTBD2 (NM_017797) sequences were identified and verified by BLAST analysis for their specificity to BTBD1 and BTBD2, respectively. The pSIREN-BTBD1 (D1\#3) shRNA vector contained the BTBD1-specific target sequence CACTCAAAGGTCCAGATTC, and the pSIREN-BTBD2 (D2\#7) shRNA vector contained the BTBD2-specific target sequence AGGTCATCTTCTACACCTA. Oligonucleotides used to 


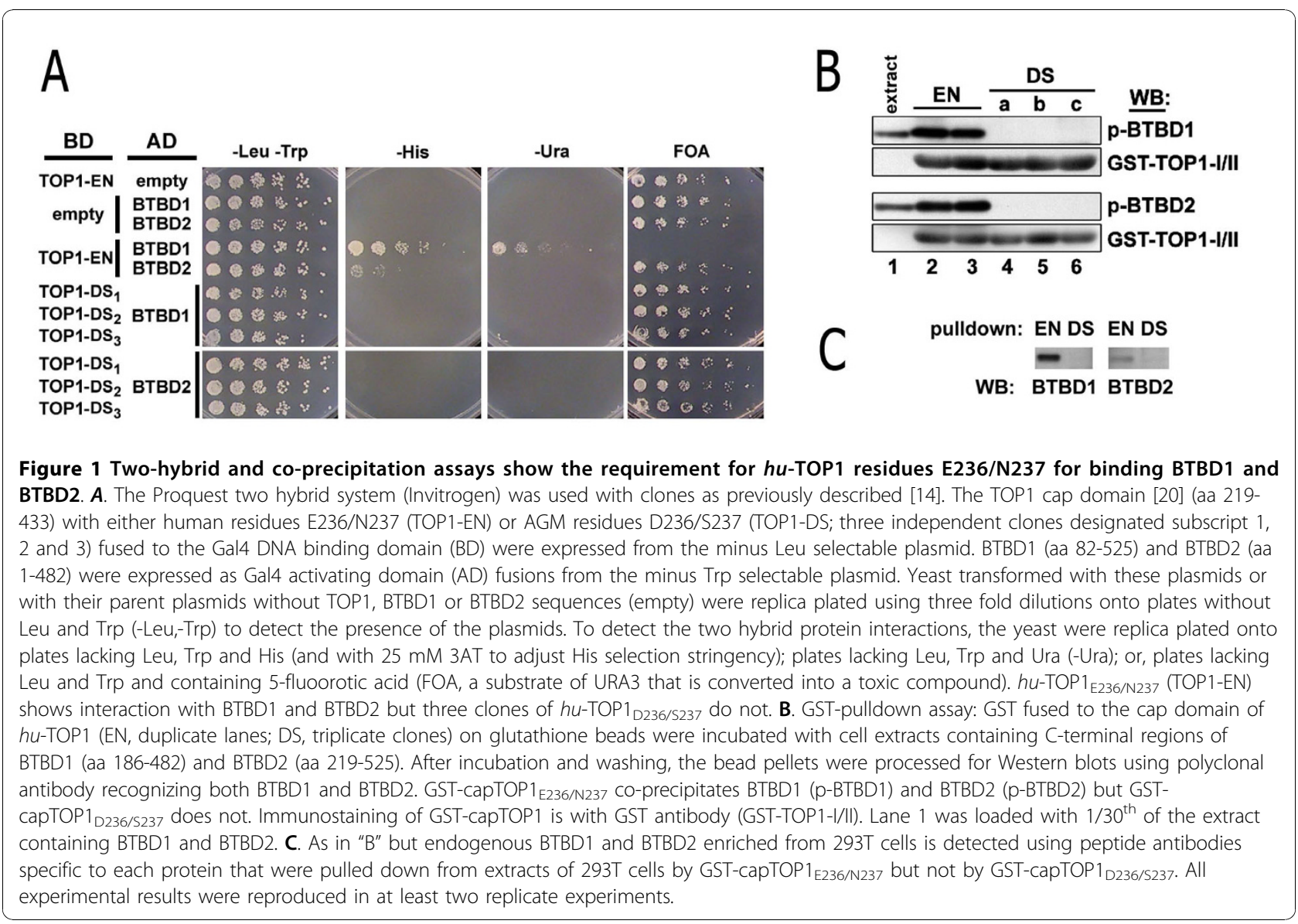

construct the negative control (S-ve) and luciferase (S-luc) shRNA vectors were supplied by the manufacturer. ShRNA vectors were constructed by inserting the annealed oligonucleotides into the linearized pSIRENRetroQ vector. The nucleotide sequence of all clones was determined to confirm the expected insertions.

\section{Silencing of BTBD1, BTBD2 and TRIM5 $\alpha$ genes}

Control (S-ve and S-luc), BTBD1 (D1\#3) and BTBD2 $(\mathrm{D} 2 \# 7)$ shRNA constructs $(16 \mu \mathrm{g})$ were transfected into $2 \times 10^{6}$ COS- 1 cells per $10 \mathrm{~cm}$ dish using Lipofectamine-2000 transfection reagent (Invitrogen, Carlsbad $\mathrm{CA})$ at a DNA $(\mu \mathrm{g})$ to Lipofectamine ${ }^{\mathrm{Tm}} 2000(\mu \mathrm{l})$ ratio of 1:2.5. After $48 \mathrm{~h}$ to $72 \mathrm{~h}$, cells expressing the shRNA constructs were harvested, tested for reduced expression of BTBD1 and BTBD2 by Northern blotting, and reseeded prior to HIV-1-luc infection.

For silencing TRIM5 $\alpha$, small-interfering RNA (siRNA) directed against TRIM5 $\alpha, 5$ '-GCCUUACGAAGUCUGAAACUU-3' [8], was used. A non-specific control siRNA, 5'-AUGAACGUGAAUUGCUCAAUU-3' [8], was included in the experiments. Both TRIM $5 \alpha$-specific and non-specific control siRNAs were purchased from Dharmacon RNA Technologies (Lafayette, CO). COS-1 cells $\left(1 \times 10^{6}\right)$ were transfected in 100-mm dishes with $15 \mathrm{nM}$ siRNA and $30 \mu \mathrm{l}$ of Lipofectamine RNAiMAX (Invitrogen, Carlsbad CA). After $48 \mathrm{~h}$ to $72 \mathrm{~h}$, cells were harvested, tested for reduced expression of TRIM $5 \alpha$ mRNA by Northern blotting, and reseeded prior to HIV-1-luc infection.

\section{RNA isolation and Northern blotting}

Cells were lysed with $1 \mathrm{ml}$ of TriPure Isolation Reagent (Roche, Indianapolis IN) and RNA was purified. Equal amounts of total RNA (10-20 $\mu \mathrm{g}$ ) were loaded for electrophoresis on a $1 \%$ formaldehyde-agarose gel and transferred to a nylon membrane. The membranes were subsequently hybridized with gene specific, ${ }^{32} \mathrm{P}$-labeled, random primed probes, and the amount of mRNA was quantified on a beta counter.

\section{Western Blot}

Cells were lysed in buffer A (50 mM Tris, pH 8.0, 150 $\mathrm{mM} \mathrm{NaCl}, 1.0 \%$ Nonidet P-40, $0.5 \%$ deoxycholate) and protein concentrations were determined using BCA kit (Pierce, Rockford IL). Equivalent amounts of protein were resolved in Tris-glycine gels, transferred to PVDF membrane, and probed with antibody generated 
against full length BTBD2 that recognizes both BTBD1 and BTBD2 (Figure 1B), peptides specific for BTBD1 or BTBD2 that do not cross-react (Figure 1C), huTOP1 (rabbit F14; LAE Biotechnology Co., Ltd., Rockville $\mathrm{MD}$ ) or GST. The blots were developed using the appropriate horseradish peroxidase-conjugated secondary antibodies and ECL reagent (Amersham, GE Health, Piscataway NJ).

\section{Reporter gene virus and Infection assays}

Vesicular stomatitis virus (VSV) G glycoprotein pseudotyped retrovirus particles containing a luciferase reporter gene were produced by cotransfecting 293T cells with the HIV-1 derived backbone plasmid (pNL4-3 env ${ }^{-} \mathrm{Vpr}^{+}$ Luc) and a plasmid for VSV-G expression (pCG). COS1 cells transfected with shRNA constructs, siRNAs or TOP1 expression constructs were seeded in 48-well plates (20,000 cells/well), incubated for 48 hours and subsequently inoculated with different amounts of VSVG pseudotyped, HIV-1 luciferase reporter gene encoding virus (VSV-G/HIV-1/Luc). Cells were lysed $48 \mathrm{~h}$ postinfection (0.1\% Triton-X-100 in PBS) and the virus infectivity was evaluated by measuring the luciferase activity of the cell lysates using the Luciferase Assay Kit (Promega, Madison WI) with a Fluoroskan Ascent luminometer (Thermo Labsystems).

\section{Results}

\section{E236 and E237 of TOP1 are required for interaction with} BTBD1 and BTBD2

In a previous study we used two hybrid and pulldown assays together with deletion fragment mapping to demonstrate the requirement for TOP1 residues 215 to 329 (15\% of the 765 aa protein) for the physical interaction with BTBD1 and BTBD2, two proteins which co-localize with TRIM5 $\delta$ [14]. This 115 amino acid fragment of TOP1 contains residues E236 and N237 previously shown by Shoya et al. to be key determinants of species-specific tropism of HIV infection. We therefore tested the requirement of these TOP1 residues for interaction with $\mathrm{BTBD} 1$ and $\mathrm{BTBD} 2$. We compared the $h u$-TOP1 core domain [20] to the core domain with residues E236 and N237 replaced with the AGM counterpart residues (E236D/N237S) for ability to interact with BTBD1 and BTBD2 using two-hybrid and GST-pulldown assays. Replacement of $h u$-TOP1 residues E236/N237 with AGM residues D236/S237 abolished the two-hybrid interactions (Figure 1A). For the pull-down assay, we fused GST to an independently folding domain of $h u$-TOP1 termed the cap domain that consists of core subdomains 1 and 2 and includes E236/N237 (GST-hu-TOP1 ${ }_{\text {cap }}$ ). This fusion protein coprecipitated purified BTBD1 and BTBD2 fragments, but
GST- $h u$-TOP $1_{\text {cap }}$ with E236D/N237S failed to co-precipitate BTBD1 or BTBD2 (Figure 1B).

Next we tested whether GST-hu-TOP $1_{\text {cap }}$ could coprecipitate endogenous BTBD1 and BTBD2 from HeLa cell extracts. Our previous efforts to detect these proteins on Western blots of whole cell lysates using antibodies generated to major portions of the proteins or to unique peptides were not successful, suggesting limited amounts of the proteins in the cells. To test whether GST- $h u$-TOP $1_{\text {cap }}$ could enrich for and co-precipitate endogenous BTBD1 and BTBD2 cellular proteins, we incubated HeLa cell extracts with GST-hu-TOP $1_{\text {cap }}$ or GST- $h u-T O P 1_{\text {cap }}$ having E236/N237 replaced with AGM residues D236/S237. GST- $h u$-TOP $1_{\text {cap }}$ co-precipitated proteins of about $60 \mathrm{kDa}$, which were recognized by antibodies specific to BTBD1 or BTBD2, respectively, and were not co-precipitated by GST-hu-TOP $1_{\text {cap }}$ with AGM residues D236/S237 (Figure 1C). Thus, the two residues of TOP1 required for enhancing the infectivity of progeny virions [7] were also required for TOP1 to physically interact with cellular BTBD1 and BTBD2.

\section{Tranfection of BTBD1 and BTBD2 shRNA silencing plasmids decreases target mRNAs and cytoplasmic bodies detected with BTBD1-specific antibody}

We investigated the potential involvement of BTBD1 and BTBD2 in restricting HIV-1 infection based on the observations that: 1) BTBD1 and BTBD2 co-localize with TRIM5 $\delta$ [13], a splice variant of TRIM5 $\alpha$; 2) $h u$ TOP1 residues E236/N237 are required for the infectivity enhancement provided to virions by the expression of $h u$-TOP1 in AGM virion-producer cells; and, 3) these same human residues are required for the physical interaction of $h u$-TOP1 with BTBD1 and BTBD2. We depleted BTBD1 or BTBD2 using RNAi. Plasmids expressing short hairpin RNA specific for BTBD1 or BTBD2 and control RNA were transfected in COS-1 African green monkey kidney cells. Northern blotting showed a $70-80 \%$ reduction of BTBD1 and BTBD2 mRNA in cells transfected with specific shRNA for BTBD1 or BTBD2, respectively, as compared to untransfected or vector controls (Figure 2A).

To evaluate the effect of RNA interference of BTBD1 and BTBD2 on their protein levels, we quantified cytoplasmic bodies stained with BTBD1-specific antibody in COS-1 cells 72 hours after transfection of the silencer plasmids. In cells transfected with only one specific silencer shRNA construct, either against BTBD1 or BTBD2, the average number of cytoplasmic bodies per cell was similar to control cells. In cells transfected with both shRNA silencer plasmids, the average number of cytoplasmic bodies per cell was reduced by $40 \%$, from $\sim 3.8$ to 2.2 (Table 1 and Figures $2 \mathrm{~B}$ and 2C). 


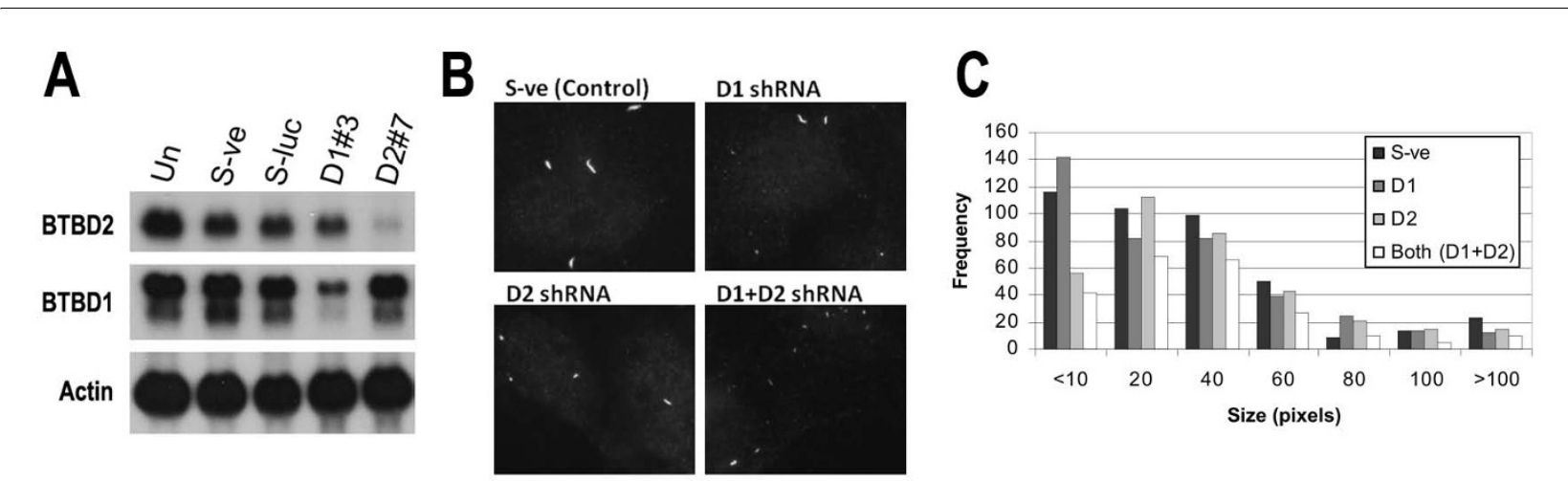

Figure 2 COS-1 cells transfected with plasmids encoding shRNAs specific to BTBD1 and BTBD2 show reductions in the specific mRNAs and cytoplasmic bodies stained with BTBD1-specific antibodies. $\boldsymbol{A}$. Northern blot shows reduced levels of expression of BTBD1 and BTBD2 mRNA in COS-1 cells transfected with BTBD1 or BTBD2 specific silencer constructs. COS-1 cells were untransfected (Un) or transfected with the negative control vector, pSIREN (S-ve), the pSIREN vector encoding Luciferase silencer (S-luc), pSIREN vector encoding BTBD1 shRNA (D1 \#3) or BTBD2 shRNA (D2 \#7). 48 hours post-transfection, transiently transfected and untransfected (COS-1) cells were harvested for RNA isolation. The Northern blot was hybridized with BTBD1 and BTBD2 specific probes. Equal amounts of RNA $(10 \mu \mathrm{g})$ were loaded onto each lane as seen with the $\beta$-actin specific probe. B. Representative immunofluorescence images showing cytoplasmic bodies in COS-1 cells transfected with pSIREN negative control vector (S-ve), silencer constructs for BTBD1 or BTBD2, or both BTBD1 and BTBD2. Seventy-two hours post-transfection cells were labeled with rabbit BTBD1-specific peptide antibody (\#4358) followed by Alexa Fluor 568 goat anti-rabbit lgG (Molecular Probes). C. Quantification of BTBD1/D2 cytoplasmic bodies. The acquired images were analyzed using TIFFany 3 (Caffeine Software) and the cytoplasmic bodies were quantified using Mathematica software (Wolfram research).

Transfection of BTBD2 shRNA silencing plasmid increases permissiveness to HIV-1 infection

We next evaluated the effect of the BTBD1 and BTBD2 shRNA plasmids transfected individually on permissiveness to HIV-1 infection in COS-1 cells. Cells transfected with the BTBD2 shRNA plasmid were 2.5 to 3 -fold more permissive to HIV-1 infection (Figure 3A). In contrast, transfection of BTBD1 shRNA did not increase permissiveness to infection (data not shown). For the sake of comparison, we used the siRNA mediated approach to deplete TRIM5 $\alpha$ in COS- 1 cells as done by others [8,21-23]. Similar to their results, siRNA targeting TRIM5 increased permissiveness to HIV-1 infection up to 20 fold (Figure 3B), which is roughly eight-fold more permissiveness than we observed in AGM cells transfected with the BTBD2 shRNA plasmid.

\section{Discussion}

Shoya et al. reported that expression of $h u$-TOP1, but not AGM-TOP1, in the AGM producer cells increased infectivity of the progeny virus five-fold [7]. Only eight amino acid residues differ between AGM-TOP1 and $h u$-TOP1. Substitution of the human residues with the AGM residues was used to identify the human residues required for the infectivity enhancement. Only replacement of glutamate-236 and asparagine-237 of $h u$-TOP1 with the corresponding conserved monkey residues (aspartate and serine, respectively) abolished the infectivity enhancement. E236 and N237 of hu-TOP1 are far from the active site (Figure 4), solvent exposed, and their replacement with the AGM counterparts (E236D/ $\mathrm{N} 237 \mathrm{~S}$ ) did not affect DNA relaxation activity (data not shown).

Shoya et al. reported that the enhanced infectivity of HIV-1 virions, provided by expression of $h u$-TOP1, but not AGM-TOP1 or E236D/N237S-hu-TOP1, was not a consequence of the amount of virus produced, the processing of Gag or Env, nor viral entry, but was associated with a four-fold greater copy number of HIV-1 DNA in human target cells. They additionally showed expression of AGM-TOP1 or E236D/N237S$h u$-TOP1 in human virion producer cells acts in a dominant negative manner to reduce the infectivity of progeny virions [7].

Table 1 shRNA interference of both BTBD1 and BTBD2 reduces the number of cytoplasmic bodies

\begin{tabular}{lcccc}
\hline & S-ve* & sh-BTBD1 & sh-BTBD2 & sh-BTBD1 + sh-BTBD2 \\
\hline TOTAL cytoplasmic bodies & 413 & 395 & 348 & 227 \\
Total cells counted & 110 & 104 & 103 & 105 \\
Cytoplasmic bodies/cell & 3.8 & 3.8 & 3.4 & 2.2 \\
\hline
\end{tabular}

* vector control 


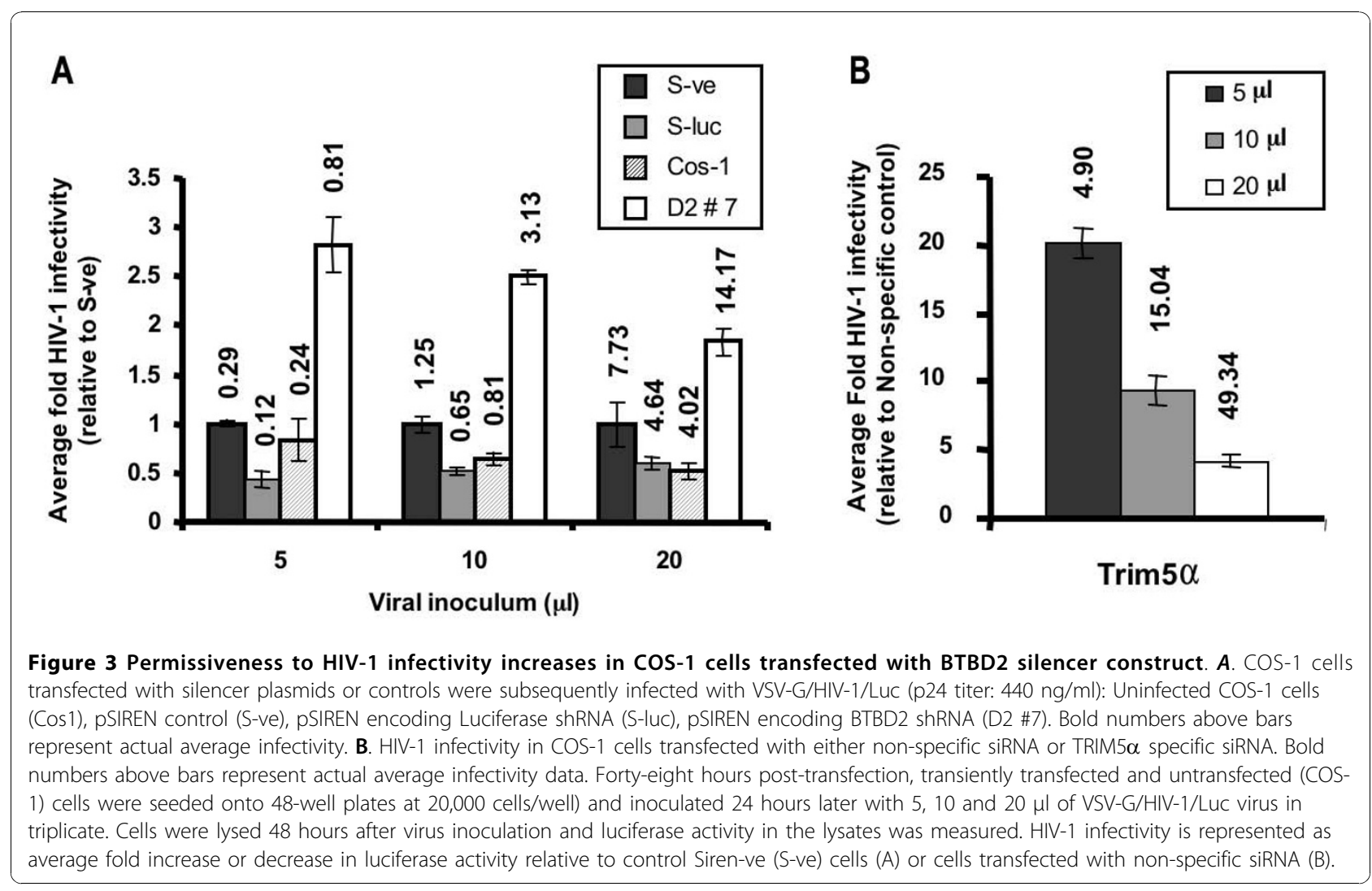

E236 and N237 are located within a fragment of $h u$-TOP 1 comprising $15 \%$ of the protein previously shown to be required for binding BTBD1 and BTBD2. Here we have shown that $h u$-TOP1 with replacements of E236 and N237 with the AGM residues D236 and S237 did not bind BTBD1 or BTBD2 in two-hybrid and GST-pulldown assays. Thus, the two residues required for $h u$-TOP1 expression in AGM producer cells to enhance the infectivity of HIV-1 virions are also required for the interaction with BTBD1 and BTBD2, two proteins which co-localize with TRIM5 $\gamma$ (unpublished) and TRIM5 $\delta$ [13].
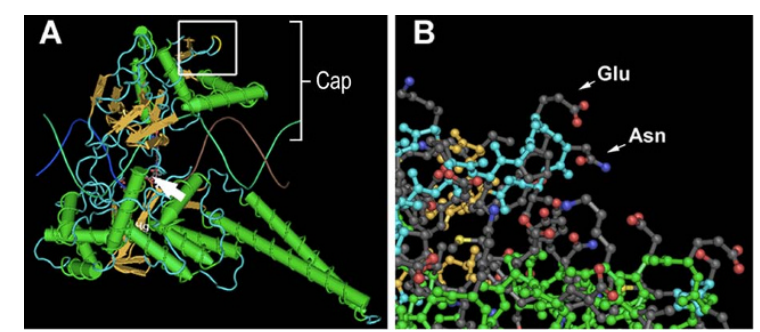

Figure 4 hu-TOP1 residues E236/N237 in crystal structure. $A$. Structure of TOP1 in covalent complex with topotecan (MMDB: 49892) shows active site (arrow), the cap region (core subdomains I and II, [20]), and residues E236 and N237 (yellow) within the box. B. Enlargement of boxed region in panel-A shows that E236 and N237 are solvent exposed.
We also found that depletion of BTBD2 in COS-1 cells increased their permissiveness to HIV-1 infection by 2.5 to 3 -fold. Similarly, in human embryonic kidney $293 \mathrm{~T}$ cells, depletion of BTBD2 increased permissiveness to infection by 1.8 to 2.8 -fold (data not shown). For comparison, TRIM5 $\alpha$ of AGM strongly restricts HIV-1 infection $[8,24,25]$ ( 20-fold in our experiments, Figure 3B), while human TRIM5 $\alpha$ only modestly inhibits HIV-1 infection by about $50 \%[8,25]$. Our data show that in both human and AGM cells, depletion of BTBD2 only modestly increased permissiveness to HIV-1 infection by about 2-3 fold. Thus, this modest restriction involving BTBD2 did not require the high level restriction provided by TRIM5 $\alpha$ of Old World primates.

BTBD1 and BTBD2 localize in cytoplasmic bodies with each other [14] and interact with each other in two hybrid assays (unpublished results). BTBD1 binds Cul3 in co-precipitation assays using bacterially purified proteins, and flag-tagged BTBD1 bound the Cul3 N-terminal 197 aa in transfected 293T cells [15]. Cul-based and other ubiquitin E3 ligases catalyze ubiquitin transfer to substrate proteins by positioning their lysines for optimal presentation to ubiquitin bound to the E2 active site [26]. Some BTB domain proteins are involved in selecting substrates for Cul3 ubiquitin ligases [15-18]. 
Our data do not rule out the speculation that the increased permissiveness to HIV-1 infection resulting from BTBD2 silencing might in some way relate to the co-localization of BTBD1 and BTBD2 with TRIM5 proteins in cytoplasmic bodies or the involvement of BTBD1 and BTBD2 with other factors that may influence permissiveness to HIV-1 infection [25]. BTBD1 and BTBD2 co-localize with TRIM5 $\delta$ and TRIM5 $\gamma$, but these variants lack the $\mathrm{C}$-terminal B30.2 domain that interacts directly with the viral capsid and determines specificity for virus restriction [27-29]. TRIM5 $\gamma$ and TRIM5 $\delta$, possibly by hetero-multimerizing with TRIM $5 \alpha$, can act dominant-negatively to inhibit the viral restricting activity of TRIM5 $\alpha[8,30]$.

\section{Conclusions}

The results presented here demonstrate that the interaction of BTBD1 and BTBD2 with TOP1 requires residues 236 and 237 of $h u$-TOP1, the same residues required for $h u$-TOP1 when expressed in AGM virion producer cells to enhance the infectivity of progeny virions. Interference with the expression of BTBD2 in either AGM or human cells increased their permissiveness to HIV-1 infectivity two- to three-fold.

\section{Acknowledgements}

The authors thank Dr. J. Sodroski and Dr. G. Meroni for TRIM5 expression plasmids.

This work was supported by grant NIH R01CA059750.

\begin{abstract}
Author details
${ }^{1}$ Department of Pathology, Uniformed Services University of the Health Sciences, 4301 Jones Bridge Road, Bethesda, MD (20814) USA. ²Department of Biochemistry and Molecular Biology, Uniformed Services University of the Health Sciences, 4301 Jones Bridge Road, Bethesda, MD (20814) USA. ${ }^{3}$ Department of Microbiology and Immunology, Uniformed Services University of the Health Sciences, 4301 Jones Bridge Road, Bethesda, MD (20814) USA. ${ }^{4}$ United States Military Cancer Institute, Uniformed Services University of the Health Sciences, 4301 Jones Bridge Road, Bethesda, MD (20814) USA
\end{abstract}

\section{Authors' contributions}

B.K. carried out shRNA knockdown, immunofluorescence and infectivity studies, and drafted portions of the manuscript. L.Z. and P.M. prepared mutant Top1 and carried out binding studies. T.S. prepared and provided VSV-G pseudotyped NL4-3 Luciferase virus particles. T.S. and C.B. provided HIV vectors, advice and consultation on infectivity assays, and edited the manuscript. M.L.C. and P.D. directed the study and wrote the manuscript. All authors read and approved the final manuscript.

\section{Competing interests}

The authors declare that they have no competing interests.

Received: 15 May 2010 Accepted: 20 November 2010 Published: 20 November 2010

\section{References}

1. Arts EJ, Wainberg MA: Human immunodeficiency virus type 1 reverse transcriptase and early events in reverse transcription. Adv Virus Res 1996, 46:97-163.

2. Freed EO: HIV-1 gag proteins: diverse functions in the virus life cycle. Virology 1998, 251(1):1-15.
3. Bieniasz PD, Grdina TA, Bogerd HP, Cullen BR: Recruitment of a protein complex containing Tat and cyclin T1 to TAR governs the species specificity of HIV-1 Tat. Embo J 1998, 17(23):7056-7065.

4. Fujinaga K, Cujec TP, Peng J, Garriga J, Price DH, Grana X, Peterlin BM: The ability of positive transcription elongation factor $B$ to transactivate human immunodeficiency virus transcription depends on a functional kinase domain, cyclin T1, and Tat. J Virol 1998, 72(9):7154-7159.

5. Garrus JE, von Schwedler UK, Pornillos OW, Morham SG, Zavitz KH, Wang HE, Wettstein DA, Stray KM, Cote M, Rich RL, et al: Tsg101 and the vacuolar protein sorting pathway are essential for HIV-1 budding. Cell 2001, 107(1):55-65.

6. Neville M, Stutz F, Lee L, Davis LI, Rosbash M: The importin-beta family member $\mathrm{Crm} 1 \mathrm{p}$ bridges the interaction between Rev and the nuclear pore complex during nuclear export. Curr Biol 1997, 7(10):767-775.

7. Shoya Y, Tokunaga K, Sawa H, Maeda M, Ueno T, Yoshikawa T, Hasegawa H, Sata T, Kurata T, Hall WW, et al: Human topoisomerase I promotes HIV-1 proviral DNA synthesis: implications for the species specificity and cellular tropism of HIV-1 infection. Proc Natl Acad Sci USA 2003, 100(14):8442-8447.

8. Stremlau M, Owens CM, Perron MJ, Kiessling M, Autissier P, Sodroski J: The cytoplasmic body component TRIM5alpha restricts HIV-1 infection in Old World monkeys. Nature 2004, 427(6977):848-853.

9. Wei P, Garber ME, Fang SM, Fischer WH, Jones KA: A novel CDK9associated C-type cyclin interacts directly with HIV-1 Tat and mediates its high-affinity, loop-specific binding to TAR RNA. Cell 1998, 92(4):451-462.

10. Zimmerman $C$, Klein KC, Kiser PK, Singh AR, Firestein BL, Riba SC, Lingappa JR: Identification of a host protein essential for assembly of immature HIV-1 capsids. Nature 2002, 415(6867):88-92.

11. Jardine D, Tachedjian G, Locarnini S, Birch C: Cellular topoisomerase I activity associated with HIV-1. AIDS Res Hum Retroviruses 1993, 9(12):1245-1250.

12. Reymond A, Meroni G, Fantozzi A, Merla G, Cairo S, Luzi L, Riganelli D, Zanaria E, Messali S, Cainarca S, et al: The tripartite motif family identifies cell compartments. Embo J 2001, 20(9):2140-2151.

13. Xu L, Yang L, Moitra PK, Hashimoto K, Rallabhandi P, Kaul S, Meroni G, Jensen JP, Weissman AM, D'Arpa P: BTBD1 and BTBD2 colocalize to cytoplasmic bodies with the RBCC/tripartite motif protein, TRIM5delta. Exp Cell Res 2003, 288(1):84-93.

14. Xu L, Yang L, Hashimoto $K$, Anderson M, Kohlhagen G, Pommier $Y$, D'Arpa P: Characterization of BTBD1 and BTBD2, two similar BTB-domaincontaining Kelch-like proteins that interact with Topoisomerase I. BMC Genomics 2002, 3(1):1.

15. Furukawa M, He YJ, Borchers $C$, Xiong Y: Targeting of protein ubiquitination by BTB-Cullin 3-Roc1 ubiquitin ligases. Nat Cell Biol 2003, 5(11):1001-1007.

16. Kobayashi A, Kang Ml, Okawa H, Ohtsuji M, Zenke Y, Chiba T, Igarashi K, Yamamoto M: Oxidative stress sensor Keap1 functions as an adaptor for Cul3-based E3 ligase to regulate proteasomal degradation of Nrf2. Mol Cell Biol 2004, 24(16):7130-7139.

17. Xu L, Wei Y, Reboul J, Vaglio P, Shin TH, Vidal M, Elledge SJ, Harper JW: BTB proteins are substrate-specific adaptors in an SCF-like modular ubiquitin ligase containing CUL-3. Nature 2003, 425(6955):316-321.

18. Zhang $Q$, Zhang $L$, Wang $B$, Ou CY, Chien $C T$, Jiang J: A hedgehoginduced BTB protein modulates hedgehog signaling by degrading $\mathrm{Ci} / \mathrm{Gli}$ transcription factor. Dev Cell 2006, 10(6):719-729.

19. Rigaut G, Shevchenko A, Rutz B, Wilm M, Mann M, Seraphin B: A generic protein purification method for protein complex characterization and proteome exploration. Nat Biotechnol 1999, 17(10):1030-1032.

20. Redinbo MR, Stewart L, Kuhn P, Champoux JJ, Hol WG: Crystal structures of human topoisomerase I in covalent and noncovalent complexes with DNA. Science 1998, 279(5356):1504-1513.

21. Hatziioannou T, Perez-Caballero D, Yang A, Cowan S, Bieniasz PD: Retrovirus resistance factors Ref1 and Lv1 are species-specific variants of TRIM5alpha. Proc Natl Acad Sci USA 2004, 101(29):10774-10779.

22. Yap MW, Nisole S, Lynch C, Stoye JP: Trim5alpha protein restricts both HIV-1 and murine leukemia virus. Proc Natl Acad Sci USA 2004, 101(29):10786-10791.

23. Nakayama EE, Miyoshi $H$, Nagai $Y$, Shioda $T$ : A specific region of 37 amino acid residues in the SPRY (B30.2) domain of African green monkey 
TRIM5alpha determines species-specific restriction of simian immunodeficiency virus SIVmac infection. J Virol 2005, 79(14):8870-8877.

24. Towers GJ: The control of viral infection by tripartite motif proteins and cyclophilin A. Retrovirology 2007, 4:40.

25. Battivelli E, Lecossier D, Matsuoka S, Migraine J, Clavel F, Hance AJ: Strainspecific differences in the impact of human TRIM5\{alpha\}, different TRIM5\{alpha\} alleles, and the inhibition of capsid-cyclophilin A interactions on the infectivity of HIV-1. J Virol 2010, 84(21):11010-11019.

26. Wu G, Xu G, Schulman BA, Jeffrey PD, Harper JW, Pavletich NP: Structure of a beta-TrCP1-Skp1-beta-catenin complex: destruction motif binding and lysine specificity of the SCF(beta-TrCP1) ubiquitin ligase. Mol Cell 2003, 11(6):1445-1456.

27. Perez-Caballero D, Hatziioannou T, Yang A, Cowan S, Bieniasz PD: Human tripartite motif 5 alpha domains responsible for retrovirus restriction activity and specificity. J Virol 2005, 79(14):8969-8978.

28. Stremlau M, Perron M, Welikala S, Sodroski J: Species-specific variation in the B30.2(SPRY) domain of TRIM5alpha determines the potency of human immunodeficiency virus restriction. J Virol 2005, 79(5):3139-3145.

29. Yap MW, Nisole S, Stoye JP: A single amino acid change in the SPRY domain of human Trim5alpha leads to HIV-1 restriction. Curr Biol 2005, 15(1):73-78.

30. Passerini LD, Keckesova Z, Towers GJ: Retroviral restriction factors Fv1 and TRIM5alpha act independently and can compete for incoming virus before reverse transcription. J Virol 2006, 80(5):2100-2105.

doi:10.1186/1743-422X-7-332

Cite this article as: Khurana et al: Human TOP1 residues implicated in species specificity of HIV-1 infection are required for interaction with BTBD2, and RNAi of BTBD2 in old world monkey and human cells increases permissiveness to HIV-1 infection. Virology Journal 2010 7:332.

\section{Submit your next manuscript to BioMed Central and take full advantage of:}

- Convenient online submission

- Thorough peer review

- No space constraints or color figure charges

- Immediate publication on acceptance

- Inclusion in PubMed, CAS, Scopus and Google Scholar

- Research which is freely available for redistribution

Submit your manuscript at www.biomedcentral.com/submit 